\title{
New Hexatic Liquid Phase Observed in Lyotropic Thin Films
}

\author{
Chih-Yu Chao, ${ }^{1, *}$ Ming-Tsong Hsu, ${ }^{1}$ Wen-Jiunn Hsieh, ${ }^{1}$ John T. Ho, ${ }^{2}$ and Ivan J. B. Lin ${ }^{3}$ \\ ${ }^{1}$ Department of Physics, National Taiwan University, Taipei 10617, Taiwan, Republic of China \\ ${ }^{2}$ Department of Physics, University at Buffalo, The State University of New York, Buffalo, New York 14260, USA \\ ${ }^{3}$ Department of Chemistry, National Dong Hwa University, Hualien 97441, Taiwan, Republic of China
}

(Received 18 August 2004; published 6 December 2004)

\begin{abstract}
An intermediate surface hexatic phase between the liquid and the crystalline phases has been found for the first time in a lyotropic lamellar liquid-crystal system. This phase is highly unusual in that it has long-range sixfold bond-orientational order but liquidlike nearest-neighbor positional correlations, and could represent a significant departure from our current understanding of defect-mediated melting in two dimensions.
\end{abstract}

DOI: 10.1103/PhysRevLett.93.247801

PACS numbers: 61.30.Eb, 64.70.Md

In three dimensions, the crystalline solid melts directly into the liquid via a first-order transition. In the theory of two-dimensional (2D) defect-mediated melting [1-3], Halperin and Nelson [2] predicted the existence of an intermediate hexatic phase, with long-range bondorientational order (BOO) but short-range positional order, between the 2D solid and the isotropic liquid. Such hexatic order has been found in freestanding thin films of several liquid-crystal (LC) materials and in other 2D systems $[4,5]$. The intermediate hexatic phase has also been seen on the surface of materials that do not exhibit bulk hexatic behavior [6], and therefore may not be all that rare in nature. It is generally expected theoretically [2] and observed experimentally [5] that, although the hexatic phase does not have long-range translational order, its positional correlation length should be appreciably longer than that in an ordinary liquid. Furthermore, it has long been suspected but never demonstrated in the laboratory that lyotropic lamellar bilayers that are common in biological membranes, because of their intrinsic 2D nature, may also exhibit hexatic behavior. Here we report the first experimental observation of a hexatic phase in partially hydrated freestanding lyotropic LC films. This phase is highly unusual in that it has only liquidlike nearest-neighbor positional correlations but nevertheless exhibits well-developed BOO. This unexpected finding may modify our understanding of 2D melting and stimulate further investigations.

Our experimental technique of using electron diffraction (ED) to study the structure of freestanding LC films [5] has been expanded to examining lyotropic films under variable humidity conditions [7]. A temperature-controlled hydrated specimen chamber has been designed in a Philips Tecnai electron microscope. The relative humidity (RH) can be controlled by means of an external water reservoir which is connected to the specimen chamber, and the water vapor pressure is adjusted by controlling the temperature of the external water reservoir. The material used is an amphiphilic 1-dodecylimi- dazolium nitrate salt $\left(\left[\mathrm{C}_{12} \mathrm{H}_{25}-\mathrm{imH}\right]\left[\mathrm{NO}_{3}\right]\right)$, which is known to exhibit lyotropic LC properties in water [8]. Prior to drawing the oriented films, the nitrate salt was mixed with about $30 \%$ distilled water to form a thin paste, which was then evenly placed around the edge of the hole in the specimen film holder. The films could be drawn only with such a mixture in the $L_{\alpha}$ phase. This was done at about $32{ }^{\circ} \mathrm{C}$ in a humidity box. The films chosen in this study contained typically a few bilayers, and appeared black under white light illumination. Their thickness was determined using optical reflectivity [9]. The wet sample was transferred to the inner chamber of the electron microscope using a humidified transfer tube. The inner chamber was then sealed off, and the pressure was pumped down to the desired water vapor pressure for the corresponding temperature. From our experience, this is the best procedure to use to ensure that the film neither breaks nor thins. Thin lyotropic smectic films were drawn and cooled down from $30^{\circ} \mathrm{C}$ for an ED measurement while maintaining a $\mathrm{RH}$ of about $90 \%$. The films ruptured when the RH was reduced to below 75\%. The ED exposure time was typically a few seconds with an electron beam current of about $0.15 \mathrm{e} / \AA^{2} \mathrm{~s}$, which had been shown not to cause significant radiation damage in similar samples [7].

Most of the results here refer to a four-bilayer film, but films with thickness of up to eight bilayers were studied. Above $25^{\circ} \mathrm{C}$, the film was in the smectic- $A L_{\alpha}$ phase. Its ED pattern, as seen in Fig. 1(a), showed a diffuse ring of constant intensity, indicating the liquid nature of this phase. When the film was cooled to below $25^{\circ} \mathrm{C}$, six symmetric diffuse arcs coexisting with the diffuse ring appeared, shown in Fig. 1(b), signifying the occurrence of long-range $\mathrm{BOO}$ on the surface bilayers while the interior remained in the $L_{\alpha}$ phase. This is very similar to the smectic- $A$-hexatic- $B$ surface-freezing transition observed in thermotropic LC thin films [10-12], in which a more ordered surface phase is found to grow onto a less ordered interior in a layer-by-layer manner. Subsequently, 

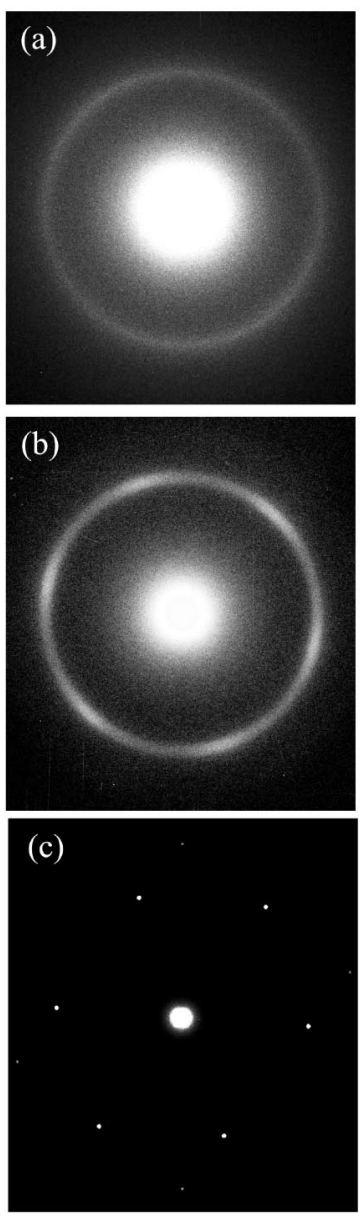

FIG. 1. Electron diffraction pattern from a four-bilayer ionic lyotropic LC film at (a) $27.5^{\circ} \mathrm{C}$, (b) $21^{\circ} \mathrm{C}$, and (c) $18.4{ }^{\circ} \mathrm{C}$ under $90 \% \mathrm{RH}$.

upon further cooling, the entire film underwent another transition at about $18.5^{\circ} \mathrm{C}$ to an orthorhombic crystalline phase, which we call $L_{E}$, as shown in Fig. 1(c). The crystalline spots are what would be expected from the herringbone crystal- $E$ structure [13]. The entire transition sequence reported here is summarized in Fig. 2.

The intermediate surface phase, which we call the lamellar hexatic liquid $\left(L_{\mathrm{hex}}\right)$, appears to be a novel lyotropic LC phase. Its integrated ED intensity suggests that the surface hexatic phase always has a thickness of one bilayer in films of different thickness. However, we did not observe the subsequent layer-by-layer transitions that

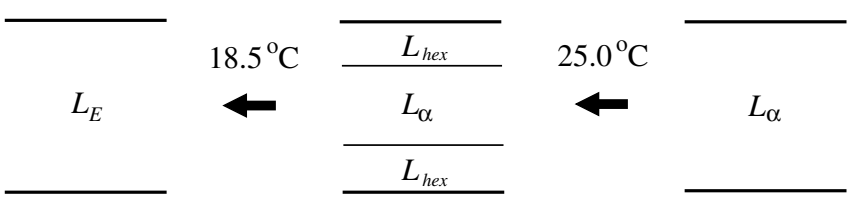

FIG. 2. Schematic representation of the phase sequence in a four-bilayer lyotropic LC film upon cooling. are prevalent in thermotropic LC films [10-12]. The sixfold azimuthal intensity modulation of this $L_{\text {hex }}$ phase denotes long-range $\mathrm{BOO}$ and can be analyzed to extract the $\mathrm{BOO}$ parameter $\mathrm{C}_{6}$ [14]. The temperature dependence of $\mathrm{C}_{6}$ at $90 \% \mathrm{RH}$ is shown in Fig. 3(a), indicating a steady and substantial increase of $\mathrm{C}_{6}$ from 0 to over 0.5 just before the transition to the crystalline phase, similar to that seen in a typical thermotropic hexatic LC phase [5]. The radial width of the $L_{\text {hex }}$ phase, however, is quite unusual, which is apparent even from a visual inspection of Fig. 1. The six arcs due to the surface phase appear to be as diffuse in the radial direction as the uniform ring due to the interior liquid, suggesting a close similarity in their positional correlations. This can be seen more quantitatively in two respects. First, in fitting the radial intensity across the six arcs, we have found that a liquidlike Lorentzian function was significantly superior to the square-root Lorentzian (SRL) function. This is in contrast to previous experiments on hexatic LC phases [5], in which it was necessary to use the SRL fit as suggested theoretically by the coupling between the hexatic and positional orders [15]. Second, the values of the positional correlation length $\xi$ in the $L_{\text {hex }}$ phase obtained from the Lorentzian fits are significantly less than those found in other hexatic LC phases [5]. Figure 4 shows the radial scans and Lorentzian fits across the surface $L_{\text {hex }}$ arcs and the interior smectic- $A L_{\alpha}$ ring at $21{ }^{\circ} \mathrm{C}$, yielding comparable $\xi$ values of 15.1 and $11.9 \AA$, respectively. The

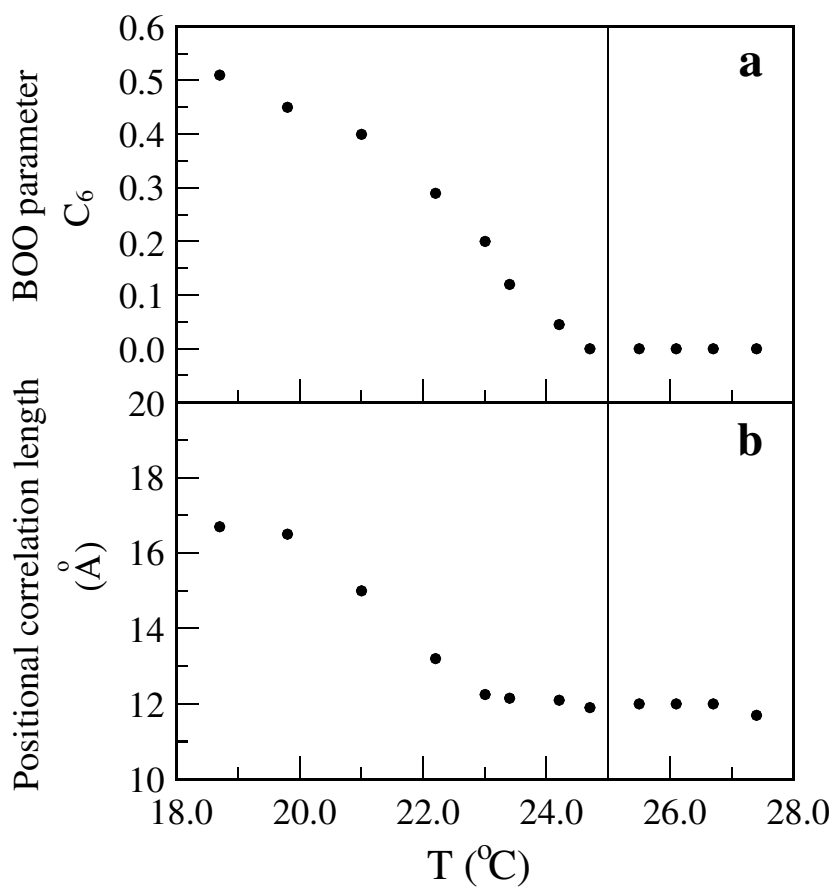

FIG. 3. Temperature dependence of (a) the BOO parameter $\mathrm{C}_{6}$ and (b) the in-plane positional correlation length $\xi$ of the surface $L_{\text {hex }}$ phase. 


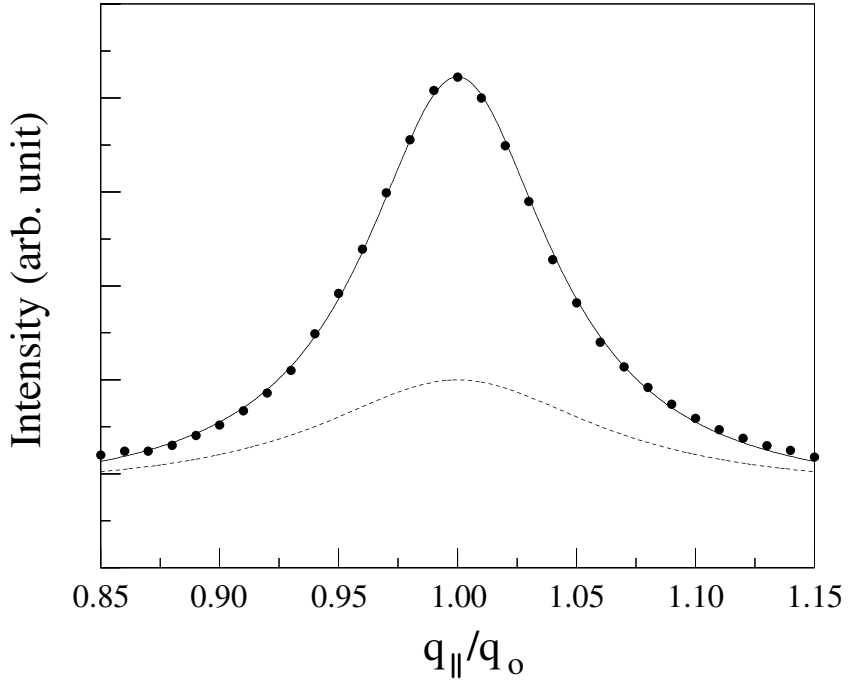

FIG. 4. Radial scan (solid circles) and Lorentzian fit (solid line) across the surface $L_{\mathrm{hex}}$ arcs at $21^{\circ} \mathrm{C}$, yielding a positional correlation length $\xi$ of $15.1 \AA$. The fit (dotted line) across the interior smectic- $A L_{\alpha}$ ring at the same temperature with a $\xi$ of $11.9 \AA$ is shown for comparison. The in-plane wave vector $q_{\|}$is normalized with respect to the intermolecular wave vector $q_{\circ}$.

temperature dependence of $\xi$ in the $L_{\text {hex }}$ phase is seen in Fig. 3(b), showing that, even when $\mathrm{C}_{6}$ has reached a significant value of $0.5, \xi$ increases only slightly (to $<17 \AA$ ) from its value of $12 \AA$ in the liquid phase. Since our ED data indicate an average intermolecular distance of $10.1 \AA$, which remains unchanged with decreasing RH or temperature, the positional correlations in the $L_{\text {hex }}$ phase appear to never extend appreciably beyond nearest neighbors and are decidedly liquidlike.

This represents the first experimental observation of hexatic behavior in a lyotropic LC system. The only previous suggestion to date of possible hexatic behavior in a lyotropic system [16] lacked direct evidence for the existence of BOO. More importantly, the $L_{\text {hex }}$ phase seen here appears to be quite different from the hexatic phases that have been observed experimentally or predicted in the theory of 2D melting. We expect the $L_{\text {hex }}$ surface to be a good model system to examine $2 \mathrm{D}$ behavior because of the fairly weak coupling between bilayers [17]. Furthermore, previous experiments on thermotropic LC films that undergo similar surface transitions show a typical interlayer penetration depth of less than one layer [12]. According to the defect-mediated mechanism of 2D melting [2], the occurrence of a relatively small number of free dislocations in the hexatic phase destroys longrange translational order but not long-range BOO. The positional correlation length $\xi$ is thus related to the density of defects. It is generally expected and also found experimentally in colloidal systems [18] that the density of defects deep in the hexatic phase is rather low, resulting in $\xi$ being significantly larger than that in an ordinary liquid. An alternative reasoning for an enhanced $\xi$ in the hexatic phase is that, because of the coupling between the translational and bond-orientational orders, we expect $\xi$ to increase as the BOO is developed [15]. This has been confirmed in direct measurements in thermotropic LC systems, which show $\xi$ increasing with decreasing temperature in the hexatic phase to values that are significantly larger than that in the smectic- $A$ liquid phase [5]. In contrast, the $L_{\text {hex }}$ phase reported in this work exhibits the unexpected coexistence of well-developed BOO and liquidlike positional correlations (or a high density of defects). Thus our result suggests a significant departure from our current understanding of the mechanism for 2D melting, which warrants further theoretical and experimental investigations.

The fact that this discovery occurs in a lyotropic system with a lamellar bilayer structure whose alkyl chains have an interdigitated arrangement in the bulk [8] leads us to suggest a possible role played by the hydration force [17]. Since the diameter of the head group of the nitrate salt molecule is about $5 \AA$ while the actual in-plane distance between molecules is twice that, there could be at least two water molecules intercalated in between the hydrated nitrate ion and imidazole core on the two ends of adjacent molecules, resulting in an additional in-plane short-range repulsive hydration force, which has been estimated in other lyotropic systems to have a range of about one water molecule $(\sim 2.5 \AA)$ beyond the primary water shell [19]. Because of the presence of several competing forces within the lamellar bilayer, the existing 2D melting theory might not be adequate to describe the lyotropic thin film system.

In summary, we have observed for the first time in a lyotropic system a surface phase with hexatic behavior, which has the unusual combination of long-range orientational order but liquidlike nearest-neighbor positional correlations.

We are grateful to T. C. Pan, K. H. Chen, Y. R. Hsu, Y. C. Shen, and C. K. Lee for participation in the early phase of this work. This work was supported by grants from the National Science Council, Taiwan, ROC.

*To whom all correspondence should be addressed. Electronic address: cychao@ntu.edu.tw

[1] J. M. Kosterlitz and D. J. Thouless, J. Phys. C 6, 1181 (1973).

[2] B. I. Halperin and D. R. Nelson, Phys. Rev. Lett. 41, 121 (1978).

[3] A. P. Young, Phys. Rev. B 19, 1855 (1979).

[4] W. H. de Jeu, B. I. Ostrovskii, and A. N. Shalaginov, Rev. Mod. Phys. 75, 181 (2003).

[5] C. F. Chou, A. J. Jin, S. W. Hui, C. C. Huang, and J. T. Ho, Science 280, 1424 (1998), and references therein. 
[6] C. Y. Chao, C. F. Chou, J. T. Ho, S. W. Hui, A. Jin, and C. C. Huang, Phys. Rev. Lett. 77, 2750 (1996).

[7] S.W. Hui, G. G. Hausner, and D. F. Parsons, J. Phys. E 9, 69 (1976).

[8] C. K. Lee, H.W. Huang, and I. J. B. Lin, Chem. Commun. 19, 1911 (2000).

[9] H. T. Chiang, V. S. Chenwhite, R. Pindak, and M. Seul, J. Phys. II (France) 5, 835 (1995).

[10] R. Geer, T. Stoebe, C. C. Huang, R. Pindak, J.W. Goodby, M. Cheng, J. T. Ho, and S.W. Hui, Nature (London) 355 , 152 (1992).

[11] A. J. Jin, M. Veum, T. Stoebe, C. F. Chou, J. T. Ho, S.W. Hui, V. Surendranath, and C. C. Huang, Phys. Rev. Lett. 74, 4863 (1995).

[12] C. Y. Chao, C. R. Lo, P. J. Wu, T.C. Pan, M. Veum, C. C. Huang, V. Surendranath, and J. T. Ho, Phys. Rev. Lett. 88, 085507 (2002).
[13] R. Geer, T. Stoebe, C. C. Huang, R. Pindak, G. Srajer, J.W. Goodby, M. Cheng, J. T. Ho, and S.W. Hui, Phys. Rev. Lett. 66, 1322 (1991).

[14] C. F. Chou, J. T. Ho, S. W. Hui, and V. Surendranath, Phys. Rev. Lett. 76, 4556 (1996).

[15] G. Aeppli and R. Bruinsma, Phys. Rev. Lett. 53, 2133 (1984).

[16] G. S. Smith, E. B. Sirota, C. R. Safinya, R. J. Plano, and N. A. Clark, J. Chem. Phys. 92, 4519 (1990).

[17] J. N. Israelachvili, Intermolecular and Surface Forces (Academic Press, London, 1992).

[18] C. A. Murray, in Bond-Orientational Order in Condensed Matter Systems, edited by K. J. Strandburg (Springer-Verlag, New York, 1992).

[19] T. J. Mclntosh and S. A. Simon, Biochemistry 32, 8374 (1993). 